\title{
Canonical quantization, path integral representations, and pseudoclassical description of massive Weyl neutrinos in external backgrounds
}

\author{
Maxim Dvornikov, ${ }^{a}$ 羽 and D. M. Gitman ${ }^{\circ} \oplus$ \\ ${ }^{a}$ Institute of Physics, University of São Paulo, CP 66318, \\ CEP 05315-970 São Paulo, SP, Brazil; \\ ${ }^{b}$ Pushkov Institute of Terrestrial Magnetism, \\ Ionosphere and Radiowave Propagation (IZMIRAN), \\ 142190 Moscow, Troitsk, Russia
}

(Dated: November 5, 2018)

\begin{abstract}
We study massive 1/2-spin particles in various external backgrounds keeping in mind applications to neutrino physics. We are mainly interested in massive Majorana (Weyl) fields. However, massive neutral Dirac particles are also considered. We formulate classical Lagrangian theory of the massive Weyl field in terms of Grassmann-odd two-component spinors. Then we construct the Hamiltonian formulation of such a theory, which turns out to be a theory with second-class constraints. Using this formulation we canonically quantize the massive free Weyl field. We derive propagators of the Weyl field and relate them to the propagator of a massive Dirac particle. We also study the massive Weyl particles propagating in the background mater. We find the path integral representation for the propagator of such a field, as well as the corresponding pseudoclassical particle action. The massless limit of the Weyl field interacting with the matter is considered and compared with results of other works. Finally, the path integral representation for the propagator of the neutral massive Dirac particle with an anomalous magnetic moment moving in the background matter and external electromagnetic field, as well as the corresponding pseudoclassical particle action are constructed.
\end{abstract}

PACS numbers: 11.10.Ef, 03.65.Pm, 14.60.Pq, 14.60.St

Keywords: massive Majorana and Dirac neutrinos; Weyl field; pseudoclassical action; background matter; external electromagnetic field

\section{INTRODUCTION}

The study of neutrinos is one of the fastest developing areas of elementary particle physics. This is mainly due to recent experimental achievements in measuring the parameters of the neutrino mixing matrix [1]. Great experimental efforts have been devoted to clarify the question of whether neutrinos are Dirac or Majorana particles (see, e.g., Ref. [2]). Nevertheless the problem of the neutrino's nature still remains open. It should be noted that the Majorana type of neutrino masses are predicted in some models of the neutrino mass generation, like the see-saw mechanism [3]. However, one cannot exclude the possibility that neutrinos will turn out to be Dirac particles. In the present work, we study, in the main (except Sec. V]), Majorana (or Weyl) fields representing neutrinos.

The first quantum field theory treatment of massive Majorana neutrinos was made in Ref. [4], where it was proposed to describe a massive Majorana field in terms of two-component Weyl spinors. Some processes involving neutrinos, like $\beta$ decay, were also discussed in Ref. [4] using Weyl field formalism. Mixed massive Majorana neutrinos possessing anomalous transition magnetic moments in an external magnetic field were studied in Ref. [5]. The treatment of massive Majorana fermions based of spinors, which belong to the nonstandard Wigner classes and violate the Lorentz invariance,

\footnotetext{
* maxim.dvornikov@usp.br

$\dagger$ gitman@if.usp.br
}

was proposed in Ref. [6]. Recently a detailed quantization of massive Majorana particles, described in terms of $c$-number Weyl fields, in vacuum and in background matter, was considered in Ref. 7].

In the present work, we continue the rigorous study of the massive Weyl field in vacuum as well as in background matter in the framework of classical and quantum theory. In Sec. III, we start with the construction of the classical Lagrangian and Hamiltonian formulations of the massive Weyl field in a vacuum using the Grassmann-valued two-component spinors. Then, based on the constructed Hamiltonian formulation, we canonically quantize this theory. We also introduce propagators of the massive Weyl field and relate them with the corresponding propagators of the massive Dirac field. The interaction of the system of spin- $1 / 2$ fermionic fields with general external fields is described in Sec.[II] Then, in Sec.[IV] we discuss the propagation of a massive Weyl field in a background matter. We find the path integral representations for all the propagators and derive the corresponding pseudoclassical actions; in particular, the pseudoclassical action for massive Weyl particles moving in a background matter. The massless limit of a Weyl field interacting with background matter is also considered. Finally, in Sec. V] we briefly discuss the massive neutral Dirac particles possessing an anomalous magnetic moment, moving in background matter and in an external electromagnetic field. We summarize the obtained results in Sec. VI. 


\section{CLASSICAL AND QUANTUM THEORY OF A FREE MASSIVE WEYL FIELD}

As we mentioned in Sec. II the most prominent candidates to be described in terms of Majorana fields are neutrinos. Now, it is a well-established fact that there are three active neutrino generations (see, e.g., Ref. [8]) and one has a nonzero mixing between them. In some theoretical models a number of sterile neutrinos is also predicted 9]. After the diagonalization of the neutrino mass matrix, one gets a number of fermionic fields which are Majorana [10]. Therefore, if we deal with Majorana particles in vacuum, we can study a single massive field without loss of generality. Our results can be straightforwardly generalized to include several neutrino generations.

A fermionic spin- $1 / 2$ field $\psi(x)$ of the mass $m$ obeys the Dirac equation:

$$
\left(\mathrm{i} \gamma^{\mu} \partial_{\mu}-m\right) \psi(x)=0, \quad x=\left(x^{\mu}\right)=\left(x^{0}, \mathbf{x}\right) .
$$

For our purposes, it convenient to chose the Dirac $\gamma$ matrices in the chiral representation:

$$
\begin{gathered}
\gamma^{\mu}=\left(\begin{array}{cc}
0 & -\sigma^{\mu} \\
-\tilde{\sigma}^{\mu} & 0
\end{array}\right), \\
\sigma^{\mu}=\left(\sigma^{0},-\boldsymbol{\sigma}\right), \quad \tilde{\sigma}^{\mu}=\left(\sigma^{0}, \boldsymbol{\sigma}\right),
\end{gathered}
$$

where $\sigma^{0}=\sigma_{0}$ is the unit $2 \times 2$ matrix, and $\boldsymbol{\sigma}$ are the Pauli matrices (see, e.g., Ref. [11]).

Let us represent the bispinor $\psi$ in terms of twocomponent Weyl spinor $\eta$ as follows:

$$
\psi=\left(\begin{array}{c}
\mathrm{i} \sigma_{2} \eta^{*} \\
\eta
\end{array}\right)
$$

Note that such a bispinor satisfies the Majorana condition $\psi^{c}=\mathrm{i} \gamma^{2} \psi^{*}=\psi$. The Dirac equation (2.1) implies the following equation for the spinor $\eta$,

$$
\sigma^{\mu} \partial_{\mu} \eta+m \sigma_{2} \eta^{*}=0
$$

see, e.g. Ref. [12].

Note that Eq. (2.4) is the Euler-Lagrange equation for the Lagrangian

$$
\mathcal{L}=\mathrm{i} \eta^{\dagger} \sigma^{\mu} \partial_{\mu} \eta-\frac{\mathrm{i}}{2} m\left(\eta^{\mathrm{T}} \sigma_{2} \eta-\eta^{\dagger} \sigma_{2} \eta^{*}\right)
$$

In this Lagrangian, we treat $\eta$ as Grassmann-odd fields [13]. In particular, due to this reason the mass term on the right-hand side of Eq. (2.5) is not zero.

In case we study massless spin- $1 / 2$ particles, the corresponding bispinor $\psi_{0}$ satisfies the equation

$$
\mathrm{i} \gamma^{\mu} \partial_{\mu} \psi_{0}=0
$$

It should be noted that there are solutions of Eq. 2.6 which satisfy the conditions $P_{\mathrm{R}} \psi_{0}=0$ (left-handed bispinors) or $P_{\mathrm{L}} \psi_{0}=0$ (right-handed bispinors), where

$$
P_{\mathrm{R}, \mathrm{L}}=\left(1 \pm \gamma^{5}\right) / 2, \quad \gamma^{5}=\mathrm{i} \gamma^{0} \gamma^{2} \gamma^{2} \gamma^{3}
$$

Considering, e.g., a left-handed bispinor, we can present it as

$$
\psi_{0}=\left(\begin{array}{c}
0 \\
\eta_{0}
\end{array}\right)
$$

Combining Eqs. (2.6) and (2.8), we get the wave equation for the spinor $\eta_{0}$,

$$
\sigma^{\mu} \partial_{\mu} \eta_{0}=0
$$

The latter equation is the Euler-Lagrange equation for the following Lagrangian:

$$
\mathcal{L}_{0}=\mathrm{i} \eta_{0}^{\dagger} \sigma^{\mu} \partial_{\mu} \eta_{0}
$$

Starting with the Lagrangian in Eq. (2.5), we find the canonical momenta as

$$
\pi=\frac{\partial_{r} \mathcal{L}}{\partial \dot{\eta}}=\mathrm{i} \eta^{*}, \quad \pi^{*}=\frac{\partial_{r} \mathcal{L}}{\partial \dot{\eta}^{*}}=0,
$$

where the subscript $r$ denotes the right derivatives; see Ref. [14]. One can easily see that the system in question has two second-class constraints:

$$
\Phi_{1}=\pi-\mathrm{i} \eta^{*}=0, \quad \Phi_{2}=\pi^{*}=0
$$

The extended Hamiltonian reads

$$
\mathcal{H}_{1}=\mathcal{H}+\Phi_{1} \lambda_{1}+\lambda_{2} \Phi_{2}
$$

where

$$
\mathcal{H}=\mathrm{i} \eta^{\dagger}(\boldsymbol{\sigma} \nabla) \eta+\frac{\mathrm{i}}{2} m\left(\eta^{\mathrm{T}} \sigma_{2} \eta-\eta^{\dagger} \sigma_{2} \eta^{*}\right)
$$

and $\lambda_{1,2}$ are Lagrange multipliers which are Grassmannodd fields. These multipliers can be found explicitly,

$$
\lambda_{1}=(\boldsymbol{\sigma} \nabla) \eta-m \sigma_{2} \eta^{*}, \quad \lambda_{2}=-\left(\boldsymbol{\nabla} \eta^{\dagger}\right) \boldsymbol{\sigma}+m \eta^{\mathrm{T}} \sigma_{2},
$$

from the conditions of the constraint conservation

$$
\left\{\Phi_{1}, \mathcal{H}_{1}\right\}=\left\{\Phi_{2}, \mathcal{H}_{1}\right\}=0
$$

where \{\} are the generalized Poisson brackets, see Ref. [14].

The presence of the second-class constraints in a system implies that in the equations of motion and in the quantization procedure, the generalized Poisson brackets must be replaced by the generalized Dirac brackets, defined as

$$
\{F, G\}_{\mathrm{D}}=\{F, G\}-\left\{F, \Phi_{i}\right\} C_{i j}\left\{\Phi_{j}, G\right\}
$$

where the matrix $\left(C_{i j}\right)$ has the components $C_{i j}=$ $\left\{\Phi_{i}, \Phi_{j}\right\}^{-1}$. In our case,

$$
C=\left(\begin{array}{ll}
0 & \mathrm{i} \\
\mathrm{i} & 0
\end{array}\right) \delta^{3}(\mathbf{x}-\mathbf{y}) .
$$


The nonzero Dirac brackets of the basis fields have the form

$$
\begin{aligned}
& \left\{\eta(\mathbf{x}, t), \eta^{*}(\mathbf{y}, t)\right\}_{\mathrm{D}}=-\mathrm{i} \delta^{3}(\mathbf{x}-\mathbf{y}), \\
& \{\eta(\mathbf{x}, t), \pi(\mathbf{y}, t)\}_{\mathrm{D}}=\delta^{3}(\mathbf{x}-\mathbf{y})
\end{aligned}
$$

Hamiltonian equations of motion have the form

$$
\begin{aligned}
& \dot{\eta}=\{\eta, \mathcal{H}\}_{\mathrm{D}}=(\boldsymbol{\sigma} \boldsymbol{\nabla}) \eta-m \sigma_{2} \eta^{*}, \\
& \dot{\eta}^{*}=\left\{\eta^{*}, \mathcal{H}\right\}_{\mathrm{D}}=\left(\boldsymbol{\sigma}^{\mathrm{T}} \boldsymbol{\nabla}\right) \eta^{*}+m \sigma_{2} \eta, \\
& \dot{\pi}=\{\pi, \mathcal{H}\}_{\mathrm{D}}=\mathrm{i}\left(\boldsymbol{\sigma}^{\mathrm{T}} \boldsymbol{\nabla}\right) \eta^{*}+\mathrm{i} m \sigma_{2} \eta \\
& \dot{\pi}^{*}=\left\{\pi^{*}, \mathcal{H}\right\}_{\mathrm{D}}=0 .
\end{aligned}
$$

Of course, one can see from Eq. (2.19) that the evolution equation for $\eta$ coincides with Eq. (2.4).

Using Eq. (2.12), it is convenient to rewrite the wave equations for $\eta$ and $\pi$ as follows:

$$
\begin{aligned}
& \dot{\eta}=(\boldsymbol{\sigma} \boldsymbol{\nabla}) \eta+\mathrm{i} \sigma_{2} m \pi, \\
& \dot{\pi}=\left(\boldsymbol{\sigma}^{\mathrm{T}} \boldsymbol{\nabla}\right) \pi+\mathrm{i} \sigma_{2} m \eta,
\end{aligned}
$$

where the operation of the complex conjugation is excluded.

The two-component Weyl spinor field $\eta$ is fermionic. When doing the canonical quantization of such a field, one should replace $\eta$ and $\pi$ with the operators $\eta \rightarrow \hat{\eta}$ and $\pi \rightarrow \hat{\pi}$, and define the equal-time anticommutators for these operators via the corresponding Dirac brackets (see Ref. [14]). The nonzero equal-time anticommutators for the basic Heisenberg operators are

$$
\begin{gathered}
{[\hat{\eta}(\mathbf{x}, t), \hat{\pi}(\mathbf{y}, t)]_{+}=\left.\mathrm{i}\{\eta(\mathbf{x}, t), \pi(\mathbf{y}, t)\}_{\mathrm{D}}\right|_{\eta=\hat{\eta}, \pi=\hat{\pi}}} \\
=\mathrm{i} \delta^{3}(\mathbf{x}-\mathbf{y}),
\end{gathered}
$$

where the fundamental Dirac bracket is given in Eq. (2.18). Equation (2.12) holds true for the corresponding operators, such that we can rewrite Eq. (2.21) as follows:

$$
\left[\hat{\eta}(t, \mathbf{x}), \hat{\eta}^{\dagger}(t, \mathbf{y})\right]_{+}=\delta^{3}(\mathbf{x}-\mathbf{y})
$$

Constructing the Heisenberg operators $\hat{\eta}(x)$ that satisfy Eq. 2.4 and the anticommutation relation [Eq. (2.22)], we obtain

$$
\begin{aligned}
\hat{\eta}(x)= & \int \frac{\mathrm{d}^{3} \mathbf{p}}{(2 \pi)^{3 / 2}} \sqrt{\frac{E+|\mathbf{p}|}{2 E}} \\
& \times\left[\left(\hat{a}_{-} w_{-}-\frac{m}{E+|\mathbf{p}|} \hat{a}_{+} w_{+}\right) e^{-\mathrm{i} p x}\right. \\
& \left.+\left(\hat{a}_{+}^{\dagger} w_{-}+\frac{m}{E+|\mathbf{p}|} \hat{a}_{-}^{\dagger} w_{+}\right) e^{\mathrm{i} p x}\right],
\end{aligned}
$$

where $E=\sqrt{|\mathbf{p}|^{2}+m^{2}}$;

$$
w_{+}=\left(\begin{array}{c}
e^{-\mathrm{i} \phi / 2} \cos \theta / 2 \\
e^{\mathrm{i} \phi / 2} \sin \theta / 2
\end{array}\right), \quad w_{-}=\left(\begin{array}{c}
-e^{-\mathrm{i} \phi / 2} \sin \theta / 2 \\
e^{\mathrm{i} \phi / 2} \cos \theta / 2
\end{array}\right)
$$

are chiral amplitudes; the angles $\phi$ and $\theta$ fix the direction of the particle momentum, $\mathbf{p}=$ $|\mathbf{p}|(\cos \phi \sin \theta, \sin \phi \sin \theta, \cos \theta) ;$ and $\hat{a}_{ \pm}^{\dagger}(\mathbf{p})$ and $\hat{a}_{ \pm}(\mathbf{p})$ are the creation and annihilation operators,

$$
\begin{aligned}
& {\left[\hat{a}_{\sigma}(\mathbf{k}), \hat{a}_{\sigma^{\prime}}^{\dagger}\left(\mathbf{k}^{\prime}\right)\right]_{+}=\delta_{\sigma \sigma^{\prime}} \delta^{3}\left(\mathbf{k}-\mathbf{k}^{\prime}\right),} \\
& {\left[\hat{a}_{\sigma}(\mathbf{k}), \hat{a}_{\sigma^{\prime}}\left(\mathbf{k}^{\prime}\right)\right]_{+}=0,} \\
& {\left[\hat{a}_{\sigma}^{\dagger}(\mathbf{k}), \hat{a}_{\sigma^{\prime}}^{\dagger}\left(\mathbf{k}^{\prime}\right)\right]_{+}=0, \quad \sigma= \pm .}
\end{aligned}
$$

Note that Eqs. (2.23) and (2.24) coincide with analogous expressions derived in Ref. [12] using heuristic arguments.

Using Eqs. (2.14), (2.23), and (2.24), we get the total energy of the massive Weyl field via the creation and annihilation operators as follows:

$$
\begin{aligned}
E_{\mathrm{tot}}= & \int \mathrm{d}^{3} \mathbf{r} \mathcal{H}=\int \mathrm{d}^{3} \mathbf{p} E\left(\hat{a}_{-}^{\dagger} \hat{a}_{-}+\hat{a}_{+}^{\dagger} \hat{a}_{+}\right) \\
& + \text {divergent terms, }
\end{aligned}
$$

where "divergent terms" contains $\delta^{3}(0)$ and can be removed by the normal ordering of operators. This result confirms the chosen interpretation of the operators $\hat{a}_{ \pm}^{\dagger}(\mathbf{p})$ and $\hat{a}_{ \pm}(\mathbf{p})$.

Let us introduce two propagators of the massive Weyl field in vacuum as follows

$$
\begin{aligned}
& S_{1}(x-y)=\mathrm{i}\left\langle 0\left|T\left\{\eta(x) \eta^{\dagger}(y)\right\}\right| 0\right\rangle, \\
& S_{2}(x-y)=-\left\langle 0\left|T\left\{\eta(x) \eta^{\mathrm{T}}(y)\right\}\right| 0\right\rangle .
\end{aligned}
$$

Here $|0\rangle$ is the vacuum vector for the annihilation operators $\hat{a}_{ \pm}(\mathbf{p})$, and $T$ is the sign of the chronological ordering. With the help of Eqs. (2.23) and (2.24), we can cast the functions $S_{1,2}$ into the following forms [12]:

$$
\begin{aligned}
& S_{1}(x)=\int \frac{\mathrm{d}^{4} p}{(2 \pi)^{4}} \frac{\tilde{\sigma}^{\mu} p_{\mu}}{m^{2}-p^{2}-\mathrm{i} \epsilon} e^{-\mathrm{i} p x}, \\
& S_{2}(x)=\int \frac{\mathrm{d}^{4} p}{(2 \pi)^{4}} \frac{\sigma_{2} m}{m^{2}-p^{2}-\mathrm{i} \epsilon} e^{-\mathrm{i} p x},
\end{aligned}
$$

where $\epsilon$ is a positive, infinitesimally small constant, and the matrices $\tilde{\sigma}^{\mu}$ are defined in Eq. (III).

For our purposes, it is convenient to relate the introduced propagators [Eq. (2.27)] to the propagator of the massive Dirac field. To proceed with the problem, let us introduce the Hamiltonian Weyl field $\Xi$ that is the pair $\pi$ and $\eta$,

$$
\Xi=\left(\begin{array}{l}
\pi \\
\eta
\end{array}\right)
$$

Using Eq. (2.20), one can find the the Hamiltonian Weyl field $\Xi$ satisfies the equation $\hat{K} \Xi=0$, where the $4 \times 4$ matrix operator $\hat{K}$ reads

$$
\hat{K}=\left(\begin{array}{cc}
\partial_{t}-\left(\boldsymbol{\sigma}^{\mathrm{T}} \boldsymbol{\nabla}\right) & -\mathrm{i} \sigma_{2} m \\
-\mathrm{i} \sigma_{2} m & \partial_{t}-(\boldsymbol{\sigma} \boldsymbol{\nabla})
\end{array}\right) .
$$


The causal propagator for the Hamiltonian field $\Xi$ is

$$
\begin{aligned}
S_{\mathrm{c}}(x-y) & =\left\langle 0\left|T\left\{\Xi(x) \Xi^{\dagger}(y)\right\}\right| 0\right\rangle, \\
\hat{K} S_{\mathrm{c}}(x) & =-\delta^{4}(x) .
\end{aligned}
$$

Introducing the function $\tilde{S}_{\mathrm{c}}$ instead of $S_{\mathrm{c}}$,

$$
\tilde{S}_{\mathrm{c}}=-\mathfrak{S}_{2} S_{\mathrm{c}} \mathfrak{S}_{2} \gamma^{0} \gamma^{5}, \quad \mathfrak{S}_{2}=\operatorname{diag}\left(\sigma_{2}, \sigma_{0}\right),
$$

we derive for this function the following equation:

$$
\left(\mathrm{i} \Gamma^{\mu} \partial_{\mu}-m \Gamma^{5}\right) \tilde{S}_{\mathrm{c}}=\delta^{4}(x),
$$

where $\Gamma^{\mu}=\Gamma^{5} \gamma^{\mu}$ and $\Gamma^{5}=-\mathrm{i} \gamma^{5}$. The $\gamma$ matrices $\Gamma^{n}=$ $\left(\Gamma^{\mu}, \Gamma^{5}\right)$ satisfy the usual $\gamma$-matrix algebra

$$
\begin{aligned}
{\left[\Gamma^{k}, \Gamma^{n}\right] } & =2 \eta^{k n}, \quad \eta_{k n}=\operatorname{diag}(1,-1, \ldots,-1), \\
k, n & =0, \ldots, 3,5,
\end{aligned}
$$

so that $\tilde{S}_{\mathrm{c}}$ can be interpreted as the causal propagator of the massive Dirac field.

The propagators of the massive Weyl field [Eq. (2.27)] can be related to the causal propagator $S_{\mathrm{c}}$ of the Hamiltonian field $\Xi$, and then to the causal propagator $\tilde{S}_{\mathrm{c}}$ of the massive Dirac field. First, we write

$$
\begin{aligned}
P_{\mathrm{L}} S_{\mathrm{c}} P_{\mathrm{L}} & =-\frac{\mathrm{i}}{2}\left(\sigma_{0}-\sigma_{3}\right) \otimes S_{1}, \\
P_{\mathrm{L}} S_{\mathrm{c}} P_{\mathrm{R}} & =\frac{\mathrm{i}}{2}\left(\sigma_{1}-\mathrm{i} \sigma_{2}\right) \otimes S_{2}, \\
\frac{1}{2}\left(\sigma_{0}-\sigma_{3}\right) \otimes S_{1} & =P_{\mathrm{L}} \tilde{S}_{\mathrm{c}} P_{\mathrm{R}} \Gamma^{0}, \\
\frac{1}{2}\left(\sigma_{1}-\mathrm{i} \sigma_{2}\right) \otimes S_{2} & =P_{\mathrm{L}} \tilde{S}_{\mathrm{c}} P_{\mathrm{L}} \Gamma^{0} \mathfrak{S}_{2},
\end{aligned}
$$

where the chiral projectors $P_{\mathrm{L}, \mathrm{R}}$ were defined in Eq. (2.7). Then, one can derive from Eqs. (2.33) and (2.34) that

$$
\begin{aligned}
& S_{1}=-\mathrm{i} \tilde{\sigma}^{\mu}\left(V_{\mu}-A_{\mu}\right), \\
& S_{2}=-\mathrm{i}\left[S-P+\frac{\mathrm{i}}{2}\left(\tilde{\sigma}^{\mu} \sigma^{\nu}-\tilde{\sigma}^{\nu} \sigma^{\mu}\right) T_{\mu \nu}\right] \sigma_{2},
\end{aligned}
$$

where $S, P, V_{\mu}, A_{\mu}$, and $T_{\mu \nu}$ are the scalar, pseudoscalar, vector, axial-vector and tensor coefficients in the expansion of $\tilde{S}_{\mathrm{c}}$ in the independent $\gamma$-matrix basis,

$$
\tilde{S}_{\mathrm{c}}=S \mathrm{I}+P \gamma^{5}+V_{\mu} \gamma^{\mu}+A_{\mu} \gamma^{5} \gamma^{\mu}+T_{\mu \nu} \sigma^{\mu \nu},
$$

where $\mathrm{I}$ is the unit $4 \times 4$ matrix and $\sigma^{\mu \nu}=(\mathrm{i} / 2)\left[\gamma^{\mu}, \gamma^{\nu}\right]_{-}$. Finally, using Eq. (2.35) we can express $S_{1,2}$ as follows:

$$
\begin{aligned}
& S_{1}=-\frac{\mathrm{i}}{4} \operatorname{tr}\left[\gamma_{\mu}\left(1-\gamma^{5}\right) \tilde{S}_{\mathrm{c}}\right] \tilde{\sigma}^{\mu}, \\
& S_{2}=-\frac{\mathrm{i}}{4} \operatorname{tr}\left[\gamma_{\mu} \gamma^{0}\left(1-\gamma^{5}\right) \tilde{S}_{\mathrm{c}}\right] \sigma^{\mu} \sigma_{2} .
\end{aligned}
$$

In Sec. IV] we shall give the expression for $\tilde{S}_{\mathrm{c}}$ in terms of the path integral.

\section{INTERACTION OF FERMIONS WITH EXTERNAL FIELDS}

In this section we briefly review the interaction of fermionic fields with external fields. We discuss the general situation when an arbitrary number of spinor fields are present. The cases of Dirac and Majorana fermions are compared. We also consider the gravitational interaction of spinor particles.

The most general classical Lagrangian describing the interaction of $1 / 2$-spin fermions $\psi_{a}, a=1,2, \ldots$, which are Grassmann-valued spinors, with a set of external fields has the form [15],

$$
\begin{aligned}
-\mathcal{L}_{\mathrm{int}}= & s_{a b} \bar{\psi}_{a} \psi_{b}+\pi_{a b} \bar{\psi}_{a} \gamma^{5} \psi_{b} \\
& +V_{a b}^{\mu} \bar{\psi}_{a} \gamma_{\mu} \psi_{b}+A_{a b}^{\mu} \bar{\psi}_{a} \gamma_{\mu} \gamma^{5} \psi_{b} \\
& +\frac{1}{2} T_{a b}^{\mu \nu} \bar{\psi}_{a} \sigma_{\mu \nu} \psi_{b}+\frac{1}{2} \Pi_{a b}^{\mu \nu} \bar{\psi}_{a} \sigma_{\mu \nu} \gamma^{5} \psi_{b},
\end{aligned}
$$

where $s_{a b}, \pi_{a b}, V_{a b}^{\mu}, A_{a b}^{\mu}, T_{a b}^{\mu \nu}$, and $\Pi_{a b}^{\mu \nu}$ are the scalar, pseudoscalar, vector, axial-vector, tensor, and pseudotensor fields, respectively. Note that these external fields can depend on spatial coordinates.

If we deal with Dirac fermions, the spinors $\psi_{a}$ and $\bar{\psi}_{a}$ are independent degrees of freedom. In this case the external fields in Eq. (3.1) are Hermitian matrices in the indices $a$ and $b$.

If we have Majorana fermions, the spinors $\psi_{a}$ and $\bar{\psi}_{a}$ are no longer independent. Instead they obey the relation, cf. Sec. II]

$$
\psi_{a}=\mathcal{C} \bar{\psi}_{a}^{\mathrm{T}}, \quad \text { or, } \quad \bar{\psi}_{a}=\psi_{a}^{\mathrm{T}} \mathcal{C},
$$

where $\mathcal{C}$ is the charge conjugation matrix which has the following properties [11]:

$$
\begin{aligned}
& \mathcal{C}=-\mathcal{C}^{-1}=-\mathcal{C}^{\mathrm{T}}=-\mathcal{C}^{\dagger}, \\
& \mathcal{C} \gamma^{\mu} \mathcal{C}^{-1}=-\gamma^{\mu \mathrm{T}}, \quad \mathcal{C} \gamma^{5} \mathcal{C}^{-1}=\gamma^{5 \mathrm{~T}}, \\
& \mathcal{C} \gamma^{\mu} \gamma^{5} \mathcal{C}^{-1}=\left(\gamma^{\mu} \gamma^{5}\right)^{\mathrm{T}}, \quad \mathcal{C} \sigma_{\mu \nu} \mathcal{C}^{-1}=-\sigma_{\mu \nu}^{\mathrm{T}},
\end{aligned}
$$

which are independent on $\gamma$-matrix representations.

Using Eqs. (3.1)-(3.3), we immediately find that for Majorana fermions the matrices $s_{a b}, \pi_{a b}, A_{a b}^{\mu}$ should be symmetric, whereas $V_{a b}^{\mu}, T_{a b}^{\mu \nu}$, and $\Pi_{a b}^{\mu \nu}$ are antisymmetric. This fact, in particular, means that the diagonal (i.e., when $a=b$ ) electromagnetic interaction of Majorana neutrinos vanishes (see also Sec. IV] below).

The interaction of a spinor particle with a gravitational field can be implemented in a locally Minkowski space by the introduction of the covariant derivative there (see, e.g., Ref. [16]). This covariant derivative also includes the contribution of the torsion, which, in principle, can have a nonzero value. The Dirac equation in flat space-time in the presence of torsion was also studied in Ref. [17]. It was shown there that the contribution of the torsion is equivalent to that ofan external axial-vector field [see Eq. [3.1)]. The issue of whether there is a difference between the gravitational interaction of Dirac and Majorana fermions was discussed in Ref. [18]. 


\section{MASSIVE WEYL NEUTRINOS IN EXTERNAL FIELDS}

Here we generalize the results of the previous section to include the interaction of the massive Weyl particles with external backgrounds. We discuss a particular case of massive neutrinos interacting with background matter and an external electromagnetic field.

Note that in Sec. II we discussed the case of a single massive Weyl field. It was also mentioned that a generalization to several mass eigenstates $\eta_{a}$ with masses $m_{a}$ is not so difficult (see, e.g., Sec. III). The most general Lagrangian which involves the interaction of the fields $\eta_{a}$ with background matter and the electromagnetic field (E, B) has the form (see, e.g., Refs. [19, 20]),

$$
\begin{aligned}
\mathcal{L}=\mathrm{i} & \eta_{a}^{\dagger} \sigma^{\mu} \partial_{\mu} \eta_{a}-\frac{\mathrm{i}}{2} m_{a}\left(\eta_{a}^{\mathrm{T}} \sigma_{2} \eta_{a}-\eta_{a}^{\dagger} \sigma_{2} \eta_{a}^{*}\right) \\
& -g_{a b}^{\mu} \eta_{a}^{\dagger} \sigma_{\mu} \eta_{b} \\
& -\frac{1}{2}\left[\mu_{a b} \eta_{a}^{\dagger} \boldsymbol{\sigma}(\mathbf{B}-\mathrm{i} \mathbf{E}) \sigma_{2} \eta_{b}^{*}\right. \\
& \left.+\left(\mu^{\dagger}\right)_{a b} \eta_{a}^{\mathrm{T}} \sigma_{2}(\mathbf{B}+\mathrm{i} \mathbf{E}) \boldsymbol{\sigma} \eta_{b}\right] \\
& -\frac{1}{2}\left[\varepsilon_{a b} \eta_{a}^{\dagger} \boldsymbol{\sigma}(\mathbf{E}+\mathrm{i} \mathbf{B}) \sigma_{2} \eta_{b}^{*}\right. \\
& \left.+\left(\varepsilon^{\dagger}\right)_{a b} \eta_{a}^{\mathrm{T}} \sigma_{2}(\mathbf{E}-\mathrm{i} \mathbf{B}) \boldsymbol{\sigma} \eta_{b}\right],
\end{aligned}
$$

where $\left(\mu_{a b}\right)$ and $\left(\varepsilon_{a b}\right)$ are the matrices of magnetic and electric dipole moments.

The interaction with background matter is characterized by the quantities $g_{a b}^{\mu}$, which are Lorentz four-vectors and Hermitian matrices in the particle species space, $g_{a b}^{\mu *}=g_{b a}^{\mu}$. As was shown in Ref. [21] (see also Sec. III), the Hermitian matrices $\left(\mu_{a b}\right)$ and $\left(\varepsilon_{a b}\right)$ must be antisymmetric.

The physical implementation of the Lagrangian in Eq. (4.1) is a system of massive Majorana neutrinos propagating in background matter under the influence of an external electromagnetic field. In case we study the system of massive neutrinos, the components of the matrix $\left(g_{a b}^{\mu}\right)$ are related to the effective potentials of the active flavor neutrinos' interaction with background matter, $U^{\mu}=\left(U^{0}, \mathbf{U}\right)=\operatorname{diag}\left(U_{\nu_{e}}^{\mu}, U_{\nu_{\mu}}^{\mu}, U_{\nu_{\tau}}^{\mu}\right)$, by means of the matrix transformation, $\left(g_{a b}^{\mu}\right)=\mathcal{U}^{\dagger} U^{\mu} \mathcal{U}$, where $\mathcal{U}$ is the mixing matrix. The zeroth component, $U^{0}$, is proportional to the effective number density of background fermions, and the spatial components, $\mathbf{U}$, are the linear combination of the matter velocity and polarization. The explicit form of $U^{\mu}$ can be found in Ref. [15].

The nonperturbative analysis of the complete system (4.1) which includes the nonzero, nondiagonal interaction with the background matter and electromagnetic field, is difficult to perform. Nevertheless, it can be carried out in frames of the perturbation theory. Thus, first, we have to study the dynamics which results from the diagonal terms of the Lagrangian in Eq. (4.1). For the sake of the simplification of notations, we shall omit the index $a: \eta \equiv \eta_{a}, g^{\mu} \equiv g_{a a}^{\mu}$, etc. Using the results of Sec. II, we obtain for the new fields an analog of wave equations in Eq. (2.20):

$$
\begin{aligned}
& \sigma_{\mu} \partial^{\mu} \eta-\mathrm{i} m \sigma_{2} \pi+\mathrm{i} g^{\mu} \sigma_{\mu} \eta=0 \\
& \sigma_{\mu}^{*} \partial^{\mu} \pi-\mathrm{i} m \sigma_{2} \eta-\mathrm{i} g^{\mu} \sigma_{\mu}^{*} \pi=0
\end{aligned}
$$

Similarly to Sec. III, we introduce the causal propagators $S_{\mathrm{c}}(x-y)=\left\langle 0\left|T\left\{\Xi(x) \Xi^{\dagger}(y)\right\}\right| 0\right\rangle$, where $\Xi^{\mathrm{T}}=(\pi, \eta)$, and the modified propagator $\tilde{S}_{\mathrm{c}}=-\mathfrak{S}_{2} S_{\mathrm{c}} \mathfrak{S}_{2} \gamma^{0} \gamma^{5}$. Using Eq. (4.2), we get the equation for the modified propagator $\tilde{S}_{\mathrm{c}}$ in the following form:

$$
\left[\Gamma^{\mu}\left(\mathrm{i} \partial_{\mu}+\mathrm{i} g_{\mu} \Gamma^{5}\right)-m \Gamma^{5}\right] \tilde{S}_{\mathrm{c}}=\delta^{4}(x)
$$

Note that Eq. (4.3) is analogous to one obtained in Ref. [17] for the propagator of a massive Dirac particle interacting with an effective torsion field $S_{\text {eff }}^{\mu}$ if we make the replacement $g^{\mu} \rightarrow-S_{\text {eff }}^{\mu}$.

Then the path integral representation for $\tilde{S}_{\mathrm{c}}$ can be found using the results of Ref. [17]. Such a representation has the form

$$
\tilde{S}_{\mathrm{c}}=\left.\exp \left(\mathrm{i} \Gamma^{n} \frac{\partial_{l}}{\partial \theta^{n}}\right) Z\left(\theta^{0}, \ldots, \theta^{5}\right)\right|_{\theta=0},
$$

where $\theta^{n}$ are Grassmann-odd variables that anticommute with $\Gamma^{n}$, and

$$
\begin{aligned}
Z= & \int_{0}^{\infty} \mathrm{d} e_{0} \int \mathrm{d} \chi_{0} \int_{e_{0}} \mathcal{M}(e) \mathrm{D} e \\
& \times \int_{x_{\mathrm{in}}}^{x_{\mathrm{out}}} \mathrm{D} x \int \mathrm{D} \tilde{\pi} \int \mathrm{D} \nu \int_{\psi(0)+\psi(1)=\theta} \mathcal{D} \psi \\
& \times \exp \left\{\mathrm{i}\left(S_{\mathrm{cl}}+S_{\mathrm{GF}}\right)+\psi_{n}(1) \psi^{n}(0)\right\} .
\end{aligned}
$$

Here

$$
\begin{aligned}
S_{\mathrm{cl}}= & \int_{0}^{1}\left[-\frac{z^{2}}{2 e}-\frac{e}{2} M^{2}+\dot{x}_{\mu} d^{\mu}\right. \\
& \left.+\mathrm{i} \chi\left(m \psi^{5}+\frac{2}{3} \psi^{\mu} d_{\mu}\right)-\mathrm{i} \psi_{n} \dot{\psi}^{n}\right] \mathrm{d} \tau \\
M^{2}= & m^{2}+g^{2}+16 \partial_{\mu} g^{\mu} \psi^{0} \psi^{1} \psi^{2} \psi^{3}, \\
z^{\mu}= & \dot{x}^{\mu}+\mathrm{i} \chi \psi^{\mu}, \quad d_{\mu}=2 \mathrm{i} \varepsilon_{\mu \nu \alpha \beta} g^{\nu} \psi^{\alpha} \psi^{\beta},
\end{aligned}
$$

where $\varepsilon_{\alpha \beta \lambda \sigma}$ is the totally antisymmetric tensor with $\varepsilon_{0123}=1$, the values $\psi^{n}$ are Grassmann-odd variables, the term

$$
S_{\mathrm{GF}}=\int_{0}^{1}[\tilde{\pi} \dot{e}+\nu \dot{\chi}] \mathrm{d} \tau
$$

is the gauge-fixing action, and the measure $\mathcal{M}(e)$ reads

$$
\mathcal{M}(e)=\int \mathcal{D} p \exp \left[\frac{\mathrm{i}}{2} \int_{0}^{1} e p^{2} \mathrm{~d} \tau\right] .
$$

In Eqs. (4.5)-(4.8), $x(\tau), p(\tau)$, and $\tilde{\pi}(\tau)$ are even trajectories, whereas $\chi(\tau)$ and $\nu(\tau)$ are odd ones and $e$ is the even variable. 
One can interpret $S_{\mathrm{cl}}$ as the parametrization-invariant pseudoclassical action of a massive neutrino moving in the background matter. It should be noted that the first example of such action was the pseudoclassical action of the massive Dirac particle in $3+1$ dimensions presented by Berezin and Marinov in Ref. [22].

It should be noted that the path integral representation of the propagator in Eqs. (4.4)-(4.8) could be very useful in calculating the propagator of a massive Weyl field in background matter since it accounts for all the loop corrections. Moreover, here we do not restrict ourselves to the studies of homogeneous matter with characteristics like number density constant in time, as was assumed in Refs. 23, 24.

It is interesting to discuss the limiting case $m \rightarrow 0$ of a massless Weyl particle propagating in background matter. Considering such a limit in Eq. (4.6), we obtain the following pseudoclassical action:

$$
\begin{aligned}
S_{\mathrm{cl}}^{(0)}= & \int_{0}^{1}\left[-\frac{1}{2 e}\left(z_{\mu}-e d_{\mu}+e g_{\mu}\right)^{2}\right. \\
& +z^{\mu} g_{\mu}-8 e \partial_{\mu} g^{\mu} \psi^{0} \psi^{1} \psi^{2} \psi^{3} \\
& \left.-\frac{\mathrm{i}}{3} \chi \psi^{\mu} d_{\mu}-\mathrm{i} \psi_{n} \dot{\psi}^{n}\right] \mathrm{d} \tau .
\end{aligned}
$$

Let us compare Eq. (4.9) with the pseudoclassical action of a massless Weyl particle proposed in Ref. [25]:

$$
\begin{aligned}
S_{\text {vac }}^{(0)}= & \int_{0}^{1}\left[-\frac{1}{2 e}\left(z_{\mu}-\varepsilon_{\mu \nu \alpha \beta} b^{\nu} \psi^{\alpha} \psi^{\beta}+\frac{\mathrm{i}}{2} \alpha b_{\mu}\right)^{2}\right. \\
& \left.-\mathrm{i} \psi_{\mu} \dot{\psi}^{\mu}\right] \mathrm{d} \tau
\end{aligned}
$$

where $\alpha$ and $b^{\mu}$ are even variables. The quantization of the action in Eq. (4.10) reproduces the quantum theory of a Weyl particle. Namely, one gets the description of a right $(\alpha=1)$ or left $(\alpha=-1)$ massless neutrino in terms of a bispinor $\Psi_{0}(x)$, which satisfies both the Dirac equation with zero mass and the Weyl condition

$$
\mathrm{i} \partial_{\mu} \gamma^{\mu} \Psi_{0}(x)=0, \quad\left(\gamma^{5}-\alpha\right) \Psi_{0}(x)=0 .
$$

On the contrary, the quantization of the action in Eq. (4.9) would recover the description of a massless $1 / 2$ spin fermion in terms of two-component Weyl spinors $\eta_{0}$ [see Eqs. (2.9) and (2.10)]. Note that some alternative pseudoclassical actions for massless fermions were discussed in Ref. [26].

Let us choose in Eq. (4.10) the gauge where $\alpha=-1$ (left neutrinos) and $b_{\mu}=2 \mathrm{ieg} g_{\mu}$. Accounting for the fact that $\psi^{5}=$ const if $m=0$, we can cast Eq. (4.9) in the form $S_{\mathrm{cl}}^{(0)}=S_{\mathrm{vac}}^{(0)}+S_{\text {int }}^{(0)}$, where

$$
\begin{aligned}
S_{\text {int }}^{(0)}= & \int_{0}^{1}\left[z^{\mu} g_{\mu}-8 e \partial_{\mu} g^{\mu} \psi^{0} \psi^{1} \psi^{2} \psi^{3}\right. \\
& \left.-\frac{\mathrm{i}}{3} \chi \psi^{\mu} d_{\mu}\right] \mathrm{d} \tau
\end{aligned}
$$

can be regarded as the pseudoclassical action that describes the interaction of massless Weyl neutrinos with the background matter.

Finally, let us discuss how to find path integral representations for the propagators of a massive Weyl field $S_{1,2}$, defined in Sec. III. For this purpose we can use Eq. (2.35). Let us decompose the generating function $Z$ in Eq. (4.12) in the variables $\theta$ :

$$
Z=\sum_{n=0}^{5} \frac{f_{i_{0} \ldots i_{n}}}{n !} \theta^{i_{0}} \cdots \theta^{i_{n}}
$$

where $f_{i_{0} \ldots i_{n}}$ are the coefficients, which are antisymmetric tensors at $n>1$. For $n=0, f_{0}=$ const, and $n=1$, the quantity $f_{n}$ is a vector in a five-dimensional space.

Using Eqs. (4.4) and (4.13), we represent $\tilde{S}_{\mathrm{c}}$ in the following form:

$$
\begin{aligned}
\tilde{S}_{\mathrm{c}} & =\sum_{n=0}^{5} \frac{\mathrm{i}^{n}}{n !} f_{i_{0} \ldots i_{n}} \Sigma^{i_{0} \ldots i_{n}}, \\
\Sigma^{i_{0} \ldots i_{n}} & =\frac{1}{n !} \Gamma^{\left[i_{0}\right.} \ldots \Gamma^{\left.i_{n}\right]} .
\end{aligned}
$$

On the basis of Eq. (4.14), we can find the coefficients in the expansion of $\tilde{S}_{\mathrm{c}}$ [Eq. (2.36)] as follows:

$$
\begin{aligned}
S & =f_{0}-\mathrm{i} f_{01235}, \quad P=f_{5}+\frac{\mathrm{i}}{4 !} \varepsilon^{\mu \nu \lambda \sigma} f_{\mu \nu \lambda \sigma}, \\
V_{\mu} & =-f_{\mu 5}-\frac{\mathrm{i}}{3 !} \varepsilon^{\alpha \beta \lambda \sigma} f_{\alpha \beta \lambda} \eta_{\sigma \mu}, \\
A_{\mu} & =f_{\mu}+\frac{\mathrm{i}}{3 !} \varepsilon^{\alpha \beta \lambda \sigma} f_{\alpha \beta \lambda 5} \eta_{\sigma \mu}, \\
T_{\mu \nu} & =\frac{1}{2}\left(\tilde{f}_{\mu \nu 5}-\mathrm{i} f_{\mu \nu}\right) \\
\tilde{f}_{\mu \nu 5} & =-\frac{1}{2} \eta_{\mu \lambda} \eta_{\nu \sigma} \varepsilon^{\lambda \sigma \alpha \beta} f_{\alpha \beta 5},
\end{aligned}
$$

where we have used the representations of the propagators $S_{1,2}$ in Eq. (2.35). Here $\eta_{\mu \nu}=\operatorname{diag}(1,-1,-1,-1)$ is the metric tensor in the Minkowski space. Then, on the basis of Eqs. (2.35) and (4.15) we can get path integral representations for $S_{1,2}$.

\section{MASSIVE DIRAC NEUTRINOS IN EXTERNAL FIELDS}

In Secs. II and IV, we studied both free massive Weyl field and the interaction of such a field with different backgrounds. The most probable implementations of such systems are massive Majorana neutrinos. However, as was mentioned in Sec. I, one cannot exclude the possibility that neutrinos are Dirac particles. That is why we consider below neutral massive Dirac particles possessing anomalous magnetic moments, which interact with background matter and with an external electromagnetic field. 
Using the results of Sec. III we can write down the most general Lagrangian which describes the interaction of several massive Dirac particles $\psi_{a}$ with matter and an electromagnetic field. On the basis of Eq. (3.1) we should identify $T_{a b}^{\mu \nu}$ with $\mu_{a b} F^{\mu \nu}$, where $\left(\mu_{a b}\right)$ are anomalous magnetic moments and $F_{\mu \nu}=(\mathbf{E}, \mathbf{B})$ is the tensor of the electromagnetic field. The interaction with background matter can be introduced if we set $V_{a b}^{\mu}=-A_{a b}^{\mu}=g_{a b}^{\mu} / 2$ in Eq. (3.1), where the quantities $g_{a b}^{\mu}$ have were already introduced in Eq. (4.1). Finally, we get this Lagrangian in the following form (see also Ref. [27] and references therein):

$$
\begin{aligned}
\mathcal{L}= & \bar{\psi}_{a}\left(\mathrm{i} \gamma^{\mu} \partial_{\mu}-m_{a}\right) \psi_{a}-g_{a b}^{\mu} \bar{\psi}_{a} \gamma_{\mu}^{\mathrm{L}} \psi_{b} \\
& -\frac{\mu_{a b}}{2} \bar{\psi}_{a} \sigma^{\mu \nu} \psi_{b} F_{\mu \nu}
\end{aligned}
$$

where $\gamma_{\mu}^{\mathrm{L}}=\gamma^{\mu}\left(1-\gamma^{5}\right) / 2$.

As we already mentioned in Sec. III, the matrix of magnetic moments, $\left(\mu_{a b}\right)$, is Hermitian for the Dirac neutrinos. It means that nonzero, diagonal elements of this matrix are possible [28]. The diagonal element $\mu_{a a}$ corresponds to a magnetic moment of the mass eigenstate $\psi_{a}$.

The exact analysis of the dynamics of the system described by the Lagrangian in Eq. (5.1) with nonzero, nondiagonal elements of the matrices $\left(g_{a b}^{\mu}\right)$ and $\left(\mu_{a b}\right)$ is complicated but can be carried out in frames of the perturbation theory. That is why, as in Sec. IV we will discuss only diagonal terms in Eq. (5.1) with $a=b$. In this case, we get the wave equation for the propagator of a single Dirac neutrino $S_{\mathrm{D}}$ :

$$
\begin{aligned}
{\left[\mathrm{i} \gamma^{\mu} \partial_{\mu}-m-\frac{1}{2} \gamma^{\mu}\left(1-\gamma^{5}\right) g_{\mu}\right.} & \\
& \left.-\frac{\mu}{2} \sigma_{\mu \nu} F^{\mu \nu}\right] S_{\mathrm{D}}(x)=-\delta^{4}(x) .
\end{aligned}
$$

where $m \equiv m_{a}, g^{\mu} \equiv g_{a a}^{\mu}$, and $\mu \equiv \mu_{a a}$. Then, using Eq. (5.2), we obtain the following equation for the modified propagator, $\tilde{S}_{\mathrm{D}}=S_{\mathrm{D}} \Gamma^{5}$ :

$$
\begin{aligned}
{\left[\Gamma ^ { \mu } \left(\mathrm{i} \partial_{\mu}-\frac{1}{2} g_{\mu}\right.\right.} & \left.+\frac{\mathrm{i}}{2} \Gamma^{5} g_{\mu}\right)-m \Gamma^{5} \\
& \left.-\frac{\mathrm{i} \mu}{2} \Gamma^{5} \Gamma^{\mu} \Gamma^{\nu} F_{\mu \nu}\right] \tilde{S}_{\mathrm{D}}(x)=\delta^{4}(x),
\end{aligned}
$$

where the matrices $\Gamma^{\mu}$ and $\Gamma^{5}$ were defined in Sec. II

One can see that the vector part of the interaction with matter is equivalent to the interaction with an effective electromagnetic field: $\left(q A^{\mu}\right)_{\mathrm{eff}}=g^{\mu} / 2$, where $q$ is an effective electric charge. The axial vector part of the interaction with matter is equivalent to the interaction with an effective torsion field: $S_{\text {eff }}^{\mu}=-g^{\mu} / 2$. Contributions due to such interactions to the propagator of the Dirac massive particle were studied in Ref. [17]. The analysis of the propagator of a fermion with an anomalous magnetic moment was made in Ref. [29].
Using the results of the latter works, we can obtain the path integral representation for the propagator $\tilde{S}_{\mathrm{D}}$. The general structure of such a representation is similar to one given by Eqs. (4.4), (4.5), (4.7), and (4.8) with the substitution of the pseudoclassical action in Eq. (4.6) by the following one:

$$
\begin{aligned}
S_{\mathrm{cl}}= & \int_{0}^{1}\left[-\frac{z^{2}}{2 e}-\frac{e}{2} M_{\mathrm{D}}^{2}-\frac{\dot{x}_{\mu}}{2}\left(g^{\mu}-d^{\mu}+8 \mathrm{i} \mu \psi^{5} F^{\mu \nu} \psi_{\nu}\right)\right. \\
& +\mathrm{i} \frac{e}{2} G_{\mu \nu} \psi^{\mu} \psi^{\nu} \\
& \left.+\mathrm{i} \chi\left(m_{\mathrm{D}}^{*} \psi^{5}+\frac{1}{3} \psi^{\mu} d_{\mu}\right)-\mathrm{i} \psi_{n} \dot{\psi}^{n}\right] \mathrm{d} \tau
\end{aligned}
$$

where

$$
\begin{aligned}
G_{\mu \nu} & =\partial_{\mu} g_{\nu}-\partial_{\nu} g_{\mu}, \\
M_{\mathrm{D}}^{2} & =m_{\mathrm{D}}^{2}+g^{2} / 4+8 \partial_{\mu} g^{\mu} \psi^{0} \psi^{1} \psi^{2} \psi^{3},
\end{aligned}
$$

and $m_{\mathrm{D}}=m-2 \mathrm{i} \mu F_{\alpha \beta} \psi^{\alpha} \psi^{\beta}$.

Again, we can mention that Eqs. (4.4), 4.5), and (5.4) are the most complete expression for a propagator of a massive neutral Dirac particle with an anomalous magnetic moment, since they exactly account for the influence of background matter and an external electromagnetic field. Besides the loop corrections, these expressions also contain the inhomogeneous matter contributions, since we do not suppose that $\partial_{\mu} g_{\nu}=0$. Thus, Eqs. (4.5), (4.4), and (5.4) generalize the results of Refs. 23, 24], where the neutrino propagator in homogeneous matter was obtained on tree level without loop corrections.

\section{CONCLUSION}

In the present work, we have studied massive $1 / 2$ spin particles in various external backgrounds, keeping in mind applications to neutrino physics. We have been mainly interested in massive Majorana (or Weyl) fields. However, massive neutral Dirac particles have also been considered. We have formulated classical Lagrangian theory of the massive Weyl field in terms of Grassmann-odd two-component spinors. Then, we have constructed the Hamiltonian formulation of such a theory, which turns out to be a theory with second-class constraints. Using this formulation, we have canonically quantized the massive free Weyl field. We have derived propagators of the Weyl field and related them to the propagator of a massive Dirac particle.

Then we have studied the massive Weyl particles propagating in a background mater. We have found the path integral representation for the propagator of the massive Weyl field in background matter, as well as we have obtained the corresponding pseudoclassical action for massive Weyl particles. The massless limit of such an action was compared with the results of other works. Finally, we have studied the path integral representations for a neutral massive Dirac particle with an anomalous magnetic moment moving in background matter under the 
influence of an external electromagnetic field. From this representation, we have derived the pseudoclassical action of the corresponding neutral massive Dirac neutrino with an anomalous magnetic moment.

The results of the present work can be applied for the study of the propagation of massive mixed (Majorana or Dirac) neutrinos in dense matter and strong electromagnetic fields. As demonstrated in Ref. [30], path integral representations of particle propagators allow one to effectively calculate the propagators. We hope that the representations derived in the present work will be helpful for describing the neutrino motion in various astrophysical and cosmological media (see, e.g., Ref. [31]).

In the case of a Dirac neutrino moving in a singular external background one should carefully define boundary conditions for corresponding solutions, as this is equivalent to choosing a self-adjoint Hamiltonian in such a background. To this end, one can use a general theory of self-adjoint extensions of symmetric operators and some analogy with the motion of charged particles in an Aharonov-Bohm field (see, e.g., Ref. [32]).

It should be noted that neutrinos may have magnetic moments (for a review, see Refs. [24, 33]). The best experimental constraint on the Dirac magnetic moment of an electron neutrino is $\mu_{\nu_{e}}<2.9 \times 10^{-11} \mu_{\mathrm{B}}$ [34], where $\mu_{\mathrm{B}}=e / 2 m_{e}$ is the Bohr magneton. Astrophysical constraints on the Dirac magnetic moments are stronger: $\mu_{\nu}<1.1 \times 10^{-12} \mu_{\mathrm{B}}$ [35]. Note that in order to sat- isfy $m_{\nu} \lesssim 1 \mathrm{eV}$, there should be a more natural scale for the Dirac magnetic moments of a neutrino: $\mu_{\nu} \lesssim$ $10^{-14} \mu_{\mathrm{B}}$ [36]. If neutrinos have magnetic moments, an inhomogeneous magnetic field, acting on such particles, can change their kinetic energy, i.e. produce work. This fact implies the possibility of the neutrino creation form the vacuum by strong inhomogeneous magnetic fields (see, e.g., Ref. [37]). In the case of Dirac neutrinos, this effects was studied in Ref. [38]. In this connection, it is interesting to generalize the technique of the present work to quantize Majorana neutrinos that have anomalous transition magnetic moments, and then to study the creation of such neutrinos by strong inhomogeneous magnetic fields. In addition, by applying similar methods, one can study the creation of Majorana neutrinos from vacuum by the inhomogeneous background matter.

\section{ACKNOWLEDGMENTS}

We are grateful to I. V. Tyutin for helpful discussions. M.D. is thankful to FAPESP for supporting his postdoctoral position. D.M.G. thanks FAPESP and $\mathrm{CNPq}$ for permanent support and the Russian Ministry of Education and Science for support under the Project 14.B37.21.0911.
[1] F. P. An et al. (Daya Bay Collaboration), Phys. Rev. Lett. 108, 171803 (2012), arXiv:1203.1669 [hep-ex]; Y. Abe et al. (Double Chooz Collaboration), Phys. Rev. Lett. 108, 131801 (2012), arXiv:1112.6353 [hep-ex]; J. K. Ahn et al. (RENO Collaboration), Phys. Rev. Lett. 108, 191802 (2012), arXiv:1204.0626 [hep-ex].

[2] M. Auger et al. (EXO Collaboration), Phys. Rev. Lett. 109, 032505 (2012), arXiv:1205.5608 [hep-ex].

[3] R. N. Mohapatra and A. Yu. Smirnov, Ann. Rev. Nucl. Part. Sci. 56, 569 (2006), hep-ph/0603118.

[4] K. M. Case, Phys. Rev. 107, 307 (1957).

[5] J. Schechter and J. W. F. Valle, Phys. Rev. D 24, 1883 (1981); 25, 283 (1982).

[6] D. V. Ahluwalia, C.-Y. Lee, and D. Schritt, Phys. Rev. D 83, 065017 (2011), arXiv:0911.2947 [hep-ph].

[7] M. Dvornikov, Found. Phys. 42, 1469 (2012), arXiv:1106.3303 [hep-th]; Nucl. Phys. B855, 760 (2012), arXiv:1108.5043 [hep-ph].

[8] J. Abdallah et al. (DELPHI Collaboration), Eur. Phys. J. C 38, 395 (2005), hep-ex/0406019 G. Mangano and P. D. Serpico, Phys. Lett. B 701, 269 (2011), arXiv:1103.1261 [astro-ph.CO].

[9] R. R. Volkas, Prog. Part. Nucl. Phys. 48, 161 (2002), hep-ph/0111326

[10] I. Yu. Kobzarev, B. V. Martem'yanov, L. B. Okun', and M. G. Shchepkin, Sov. J. Nucl. Phys. 32, 823 (1980); J. Schechter and J. W. F. Valle, Phys. Rev. D 22, 2227 (1980).

[11] C. Itzykson and J.-B. Zuber, Quantum Field Theory
(McGraw-Hill, New York, 1980), pp. 691-696.

[12] M. Fukugita and T. Yanagida, Physics of Neutrinos and Applications to Astrophysics (Springer, Berlin, 2003), pp. 289-301.

[13] F. A. Berezin, The Method of Second Quantization (Academic Press, New York, 1966).

[14] D. M. Gitman and I. V. Tyutin, Quantization of Fields with Constraints (Springer, Berlin, 1990).

[15] M. Dvornikov and A. Studenikin, J. High Energy Phys. 09 (2002) 016, hep-ph/0202113.

[16] R. T. Hammond, Rep. Prog. Phys. 65, 599 (2002).

[17] B. Geyer, D. Gitman, and I. Shapiro, Int. J. Mod. Phys. A 15, 3861 (2000), hep-th/9910180

[18] D. Singh, N. Mobed, and G. Papini, Phys. Rev. Lett. 97, 041101 (2006), gr-qc/0605153 J. F. Nieves and P. B. Pal, Phys. Rev. Lett. 98, 069001 (2007), gr-qc/0610098.

[19] B. Kayser, Phys. Rev. D 26, 1662 (1982).

[20] P. D. Mannheim, Phys. Rev. D 37, 1935 (1988).

[21] S. Pastor, J. Segura, V. B. Semikoz, and J. W. F. Valle, Nucl. Phys. B566, 92 (2000), hep-ph/9905405.

[22] F. A. Berezin and M. S. Marinov, Ann. Phys. (N.Y.) 104, 336 (1977).

[23] I. Pivovarov and A. Studenikin, Proc. Sci. HEP2005 (2005) 191, hep-ph/0512031

[24] M. Dvornikov, in: Neutrinos: Properties, Sources and Detection, ed. by J. P. Greene (Nova Science Publishers, New York, 2011), pp. 23-90, arXiv:1011.4300 [hep-ph].

[25] D. M. Gitman, A. E. Gonçalves, and I. V. Tyutin. Phys. Rev. D 50, 5439 (1994), hep-th/9409187. 
[26] J. L. Cortes and M. S. Plyushchay, Comment on "New pseudoclassical model for Weyl particles", hep-th/9602106 K. S. Nirov and M. S. Plyushchay, Phys. Lett. B 405, 114 (1997), hep-th/9707070 P. Howe, S. Penati, M. Pernici, and P. Townsend, Phys. Lett. B 215555 (1988); P. Howe, S. Penati, M. Pernici, and P. Townsend, Class. Quant. Grav. 6, 1125 (1989); A. Barducci, R. Casalbuoni, D. Dominici, and L. Lusanna, Phys. Lett. 100B, 126 (1981); J. L. Cortés, M. S. Plyushchay, and L. Velázquez, Phys. Lett. B 306, 34 (1993).

[27] M. Dvornikov, Phys. Atom. Nucl. 75, 227 (2012), arXiv:1008.3115 [hep-ph].

[28] J. F. Nieves, Phys. Rev. D 26, 3152 (1982).

[29] D. M. Gitman, Nucl. Phys. B488, 490 (1997), hep-th/9608180.

[30] D. M. Gitman and S. I. Zlatev, Phys. Rev. D 55, 7701 (1997), hep-th/9608179.

[31] G. G. Raffelt, Stars as Laboratories for Fundamental Physics (University of Chicago Press, Chicago, 1996); C. Giunti and C. W. Kim, Fundamentals of Neutrino Physics and Astrophysics (Oxford University Press, Oxford, 2007).

[32] D. M. Gitman, I. V. Tyutin, and B. L. Voronov, Self- adjoint Extensions in Quantum Mechanics: General Theory and Applications to Schrödinger and Dirac Equations with Singular Potentials (Birkhauser, Boston, 2012).

[33] C. Broggini, C. Giunti, and A. Studenikin, Adv. High Energy Phys. 2012, 459526 (2012), arXiv:1207.3980 [hep$\mathrm{ph}$.

[34] A. G. Beda, V. B. Brudanin, V. G. Egorov, D. V. Medvedev, V. S. Pogosov, M. V. Shirchenko, and A. S. Starostin (GEMMA Collaboration), Adv. High Energy Phys. 2012, 350150 (2012).

[35] A. V. Kuznetsov, N. V. Mikheev, and A. A. Okrugin, Int. J. Mod. Phys. A 24, 5977 (2009), arXiv:0907.2905 [hepph].

[36] N. F. Bell, V. Cirigliano, M. J. Ramsey-Musolf, P. Vogel, and M. B. Wise, Phys. Rev. Lett. 95, 151802 (2005), hep-ph/0504134

[37] E. S. Fradkin, D. M. Gitman, and Sh. M. Shvartsman, Quantum Electrodynamics with Unstable Vacuum (Springer, Berlin, 1991).

[38] S. P. Gavrilov and D. M. Gitman, Consistent approach to creation of neutral fermions with anomalous magnetic moment from vacuum by inhomogeneous magnetic field, arXiv:1211.6776 [hep-th]. 